\title{
Some Features of $\alpha$-Regular Spaces in Supra Fuzzy Topology
}

\author{
M. F. Hoque ${ }^{1 *}$, M. S. Hossain ${ }^{2}$ and D. M. Ali $^{2}$ \\ ${ }^{1}$ Department of Mathematics, Pabna Science and Technology University, Pabna-6600, Bangladesh \\ ${ }^{2}$ Department of Mathematics, Rajshahi University, Rajshahi-6205, Bangladesh
}

Received 11 September 2011, accepted in final revised form 17 April 2012

\begin{abstract}
We introduce and study supra fuzzy $\alpha$-regular spaces and we establish some relationships among them in this paper. We also study some other properties of these concepts and obtain their several features.
\end{abstract}

Keywords: Fuzzy regular spaces; Supra fuzzy regular spaces.

(c) 2012 JSR Publications. ISSN: 2070-0237 (Print); 2070-0245 (Online). All rights reserved.

doi: http://dx.doi.org/10.3329/jsr.v4i2.8157 J. Sci. Res. 4 (2), 373-382 (2012)

\section{Introduction}

The fundamental concept of a fuzzy set was introduced by Zadeh [1] in 1965 to provide a foundation for the development of many areas of knowledge. Chang [2] in 1968 and Lowen [3] in 1976 developed the theory of fuzzy topological spaces using fuzzy sets. In 1983, Mashhour [4] introduced supra topological spaces and studied s-continuous functions and $s^{*}$-continuous functions. In 1987, Abd EL-Monsef [5] introduced the fuzzy supra topological spaces and studied fuzzy supra continuous functions and characterized a number of basic concepts. A note on fuzzy regularity concepts was given by Ali [6] in 1990. In this paper, we study some features of regular spaces and obtain their certain characterizations in supra fuzzy topological spaces. As usual $I=[0,1]$ and $I_{1}=[0,1)$.

Definition 1.1 [1]: For a set $X$, a function $u: X \rightarrow[0,1]$ is called a fuzzy set in $X$. For every $x \in X, u(x)$ represents the grade of membership of $x$ in the fuzzy set $u$. Some authors say that $u$ is a fuzzy subset of $X$. Thus a usual subset of $X$, is a special type of a fuzzy set in which the range of the function is $\{0,1\}$.

Definition 1.2 [1]: Let $X$ be a nonempty set and $A$ be a subset of $X$. The function

\footnotetext{
*Corresponding author: fazlul_math@yahoo.co.in
} 


$$
1_{A}: X \rightarrow[0,1] \text { defined by } 1_{A}(x)=\left\{\begin{array}{cc}
1 & \text { if } x \in A \\
0 & \text { if } x \notin A
\end{array}\right.
$$

is called the characteristic function of $A$. We also write $1_{X}$ for the characteristic function of $\{x\}$. The characteristic functions of subsets of a set $X$ are referred to as the crisp sets in $X$.

Definition 1.3 [2]: Let $X$ be a non empty set and $t$ be the collection of fuzzy sets in $I^{X}$. Then $t$ is called a fuzzy topology on $X$ if it satisfies the following conditions:

(i) $1,0 \in t$,

(ii) If $u_{\mathrm{i}} \in t$ for each $i \in \Lambda$, then $\cup_{i \in \Lambda} u_{\mathrm{i}} \in t$.

(iii) If $u_{1}, u_{2} \in t$ then $u_{1} \cap u_{2} \in t$.

If $t$ is a fuzzy topology on $X$, then the pair $(X, t)$ is called a fuzzy topological space (fts, in short) and members of $t$ are called $t$ - open (or simply open ) fuzzy sets. If $u$ is open fuzzy set, then the fuzzy sets of the form $1-u$ are called $t$ - closed (or simply closed) fuzzy sets.

Definition 1.4 [3]: Let $X$ be a nonempty set and $t$ be a collection of fuzzy sets in $I^{X}$ such that

(i) $1,0 \in t$,

(ii) If $u_{\mathrm{i}} \in t$ for each $i \in \Lambda$, then $\cup_{i \in \Lambda} u_{\mathrm{i}} \in t$.

(iii) If $u_{1}, u_{2} \in t$ then $u_{1} \cap u_{2} \in t$.

(iv) all constant fuzzy sets in $X$ belong to $t$.

Then $t$ is called a fuzzy topology on $X$.

Definition 1.5 [4]: Let $X$ be a nonempty set. A subfamily $t^{*}$ of $I^{X}$ is said to be a supra topology on $X$ if and only if

(i) $1,0 \in t^{*}$,

(ii) If $u_{\mathrm{i}} \in t^{*}$ for each $i \in \Lambda$, then $\cup_{\mathrm{i} \in \Lambda} u_{\mathrm{i}} \in t^{*}$.

Then the pair $\left(X, t^{*}\right)$ is called a supra fuzzy topological spaces. The elements of $t^{*}$ are called supra open sets in $\left(X, t^{*}\right)$ and complement of supra open set is called supra closed set.

Example 1.6 Let $X=\{x, y\}$ and $u, v \in I^{X}$ are defined by $u(x)=0.8, u(y)=0.6$ and $v(x)=0.6, v(y)=0.8$. Then we have $w(x)=(u \cup v)(x)=0.8, w(y)=(u \cup v)(y)=0.8$ and $k(x)$ $=(u \cap v)(\mathrm{x})=0.6, k(y)=(u \cap v)(y)=0.6$. If we consider $t^{*}$ on $X$ generated by $\{0, \mathrm{u}, \mathrm{v}, \mathrm{w}, 1\}$, then $t^{*}$ is supra fuzzy topology on $X$ but $t^{*}$ is not fuzzy topology. Thus we see that every fuzzy topology is supra fuzzy topology but the converse is not always true. 
Definition 1.7 [4]: Let $(X, t)$ and $(X, s)$ be two topological spaces. Let $t^{*}$ and $s^{*}$ are associated supra topologies with $t$ and $s$ respectively and $f:\left(X, t^{*}\right) \rightarrow\left(Y, s^{*}\right)$ be a function. Then the function $f$ is a supra fuzzy continuous if the inverse image of each i.e., if for any $v \in \mathrm{s}^{*}, f^{-1}(v) \in t^{*}$. The function $f$ is called supra fuzzy homeomorphic if and only if $f$ is supra bijective and both $f$ and $f^{-1}$ are supra fuzzy continuous.

Definition 1.8 [4]: Let $\left(X, t^{*}\right)$ and $\left(Y, s^{*}\right)$ be two supra topological spaces. If $u_{1}$ and $u_{2}$ are two supra fuzzy subsets of $X$ and $Y$ respectively, then the Cartesian product $u_{1} \times u_{2}$ is a supra fuzzy subset of $X \times Y$ defined by $\left(u_{1} \times u_{2}\right)(x, y)=\min \left[u_{1}(x), u_{2}(y)\right]$, for each pair $(x, y) \in X \times Y$.

Definition 1.9[10]: Suppose $\left\{X_{\mathrm{i}}, i \in \Lambda\right.$ \}, be any collection of sets and $X$ denoted the Cartesian product of these sets, i.e., $X=\prod_{\mathrm{i} \in \Lambda} X_{\mathrm{i}}$. Here $X$ consists of all points $p=<a_{\mathrm{i}}$ , $i \in \Lambda>$, where $a_{\mathrm{i}} \in X_{\mathrm{i}}$. For each $j_{o} \in \Lambda$, we define the projection $\pi_{j o}: X \rightarrow X_{\mathrm{jo}}$ by $\pi$ jo $\left(<a_{\mathrm{i}}: i \in \Lambda>\right)=a_{\mathrm{jo}}$. These projections are used to define the product supra topology.

Definition 1.10 [10]: Let $\left\{X_{\alpha}\right\}_{\alpha \in \Lambda}$ be a family of nonempty sets. Let $X=\Pi_{\alpha \in \Lambda} X_{\alpha}$ be the usual product of $X_{\alpha}$ 's and let $\pi_{\alpha}: X \rightarrow X_{\alpha}$ be the projection. Further, assume that each $X_{\alpha}$ is a supra fuzzy topological space with supra fuzzy topology $t_{\alpha}^{*}$. Now the supra fuzzy topology generated by $\left\{\pi_{\alpha}^{-1}\left(b_{\alpha}\right): b_{\alpha} \in t^{*}{ }_{\alpha}, \alpha \in \Lambda\right\}$ as a sub basis, is called the product supra fuzzy topology on $X$. Thus if $w$ is a basis element in the product, then there exist $\alpha_{1}, \alpha_{2}, \ldots \alpha_{\mathrm{n}} \in \Lambda$ such that $w(x)=\min \left\{b_{\alpha}\left(\mathrm{x}_{\alpha}\right): \alpha=1,2,3, \ldots \ldots \mathrm{n}\right\}$, where $x$ $=\left(x_{\alpha}\right)_{\alpha \in \Lambda} \in X$.

Definition 1.11 [5]: Let $(X, T)$ be a topological space and $T^{*}$ be associated supra topology with $T$. Then a function $f: X \rightarrow R$ is lower semi continuous if and only if $\{x \in X: f(x)>\alpha\}$ is open for all $\alpha \in R$. The lower semi continuous topology on $\mathrm{X}$ associated with $\mathrm{T}^{*}$ is $\omega\left(T^{*}\right)=\{\mu: X \rightarrow[0,1], \mu$ is supra lsc $\}$. If $\omega\left(T^{*}\right):\left(X, T^{*}\right) \rightarrow[0,1]$ be the set of all lower semi continuous (lsc) functions, then we can easily show that $\omega\left(T^{*}\right)$ is a supra fuzzy topology on $\mathrm{X}$. Let $\mathrm{P}$ be the property of a supra topological space $\left(\mathrm{X}, \mathrm{T}^{*}\right)$ and FP be its supra fuzzy topological analogue. Then FP is called a 'good extension' of $\mathrm{P}$ " if and only if the statement $\left(X, T^{*}\right)$ has $\mathrm{P}$ if and only if $\left(X, \omega\left(T^{*}\right)\right)$ has $\mathrm{FP}$ ” holds good for every supra topological space $\left(X, T^{*}\right)$.

\section{2. $\alpha$-Regular Spaces in Supra Fuzzy Topology}

Definition 2.1: Let $(X, t)$ be a fuzzy topological space and $t^{*}$ be associated supra topology with $t$ and $\alpha \in I_{1}$. Then

(a) $\left(X, t^{*}\right)$ is an $\alpha$ - SFR (i) space if and only if for all $w \in\left(t^{*}\right)^{\mathrm{c}}$ with $w(x)<1, \forall$ $x \in X$, there exist $u, \mathrm{v} \in t^{*}$ such that $u(x)=1, v(y)=1, \mathrm{y} \in w^{-1}\{1\}$ and $u \cap v \leq \alpha$.

(b) $\left(X, t^{*}\right)$ is an $\alpha$-SFR(ii) space if and only if for all $w \in\left(t^{*}\right)^{\mathrm{c}}$ with $w(x)<1, \forall x \in$ $X$, there exist $u, v \in t^{*}$ such that $u(x)>\alpha, v(y)=1, y \in w^{-1}\{1\}$ and $u \cap v \leq \alpha$. 
(c) $\left(X, t^{*}\right)$ is an $\alpha$-SFR(iii) space if and only if for all $w \in\left(t^{*}\right)^{\mathrm{c}}$ with $w(x)=0, \forall x$ $\in X$, there exist $u, v \in \mathrm{t}^{*}$ such that $u(x)=1, v(y)=1, y \in w^{-1}\{1\}$ and $u \cap v \leq \alpha$.

(d) $\left(X, t^{*}\right)$ is an $\alpha$-SFR(iv) space if and only if for all $w \in\left(t^{*}\right)^{c}$ with $w(x)=0, \forall$ $x \in X$, there exist $u, v \in t^{*}$ such that $u(x)>\alpha, v(y)=1, y \in w^{-1}\{1\}, u \cap v \leq \alpha$.

Theorem 2.2: Let $(X, t)$ be a fuzzy topological space and $t^{*}$ be associated supra topology with $t$. Then the following implications are true:

(a) $\left(X, t^{*}\right)$ is $\alpha$-SFR(i) implies ( $\left.X, t^{*}\right)$ is $\alpha$ - SFR(ii) implies $\left(X, t^{*}\right)$ is $\alpha$ - SFR(iv).

(b) $\left(X, t^{*}\right)$ is $\alpha$-SFR(i) implies ( $\left.X, t^{*}\right)$ is $\alpha$ - SFR(iii) implies $\left(X, t^{*}\right)$ is $\alpha$ - SFR(iv).

Also, these can be shown in a diagram as follows:

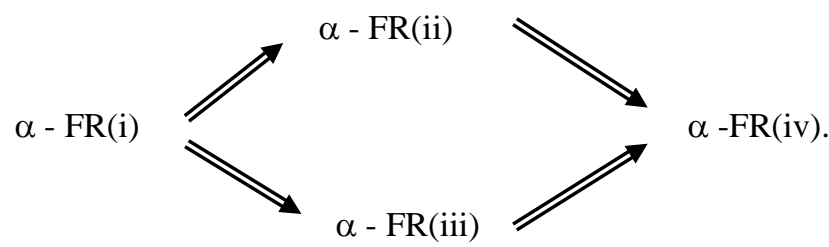

Proof. First, suppose that $\left(X, t^{*}\right)$ is $\alpha$ - SFR (i). We have to prove that $\left(X, t^{*}\right)$ is $\alpha$ - SFR(ii). Let $w \in\left(t^{*}\right)^{c}$ with $w(x)<1, x \in X$. Since $\left(X, t^{*}\right)$ is $\alpha$-SFR(i), for $\alpha \in I_{1}$, there exist $u, v \in t^{*}$ such that $u(x)=1, v(y)=1, y \in w^{-1}\{1\}$ and $u \cap v \leq \alpha$. Now, we see that $u(x)>\alpha, v(y)=1$, $y \in w^{-1}\{1\}$ and $u \cap v \leq \alpha$. Hence by definition $\left(X, t^{*}\right)$ is $\alpha$-SFR (ii).

Next, Suppose that $\left(X, t^{*}\right)$ is $\alpha$ - SFR (ii). Let $w \in\left(t^{*}\right)^{c}$ with $w(x)<1, x \in X$, then for $\alpha \in I_{1}$, there exist $u, v \in t^{*}$ such that $u(x)>1, v(y)=1, y \in w^{-1}\{1\}$ and $u \cap v \leq \alpha$. Now, we see that $u(x)>\alpha, v(y)=1, y \in w^{-1}\{1\}$ and $u \cap v \leq \alpha$. Hence by definition $\left(X, t^{*}\right)$ is $\alpha$-SFR (iv).

In the same way, we can prove that

$$
\begin{aligned}
& \left(X, t^{*}\right) \text { is } \alpha-\operatorname{SFR}(\mathrm{i}) \Rightarrow\left(X, t^{*}\right) \text { is } \alpha-\operatorname{SFR}(i i i) \\
& \left(X, t^{*}\right) \text { is } \alpha-\operatorname{SFR}(\mathrm{iii}) \Rightarrow\left(X, t^{*}\right) \text { is } \alpha-\operatorname{SFR}(i v) .
\end{aligned}
$$

Now, we give some examples to show the non implication among $\alpha$ - SFR (i), $\alpha$ - SFR (ii), $\alpha$ - SFR (iii) and $\alpha$ - SFR (iv).

Example 2.3: Let $X=\{x, y\}$ and $u, v \in I^{X}$ are defined by $u(x)=0.9, u(y)=0, v(x)=0.5$ and $v(y)=1$. Consider the supra fuzzy topology $t^{*}$ on $X$ generated by $\{0, u, v, 1$, constants $\}$. Then for $w=1-u$ and $\alpha=0.7$, we see that $\left(X, t^{*}\right)$ is $\alpha$-SFR(ii) but $\left(X, t^{*}\right)$ is not $\alpha$-SFR(i).

Example 2.4: Let $X=\{x, y\}$ and $u, v \in I^{X}$ are defined by $u(x)=0.2, u(y)=0.3, v(x)=0.3$, $v(y)=0.2$. Consider the supra fuzzy topology $t^{*}$ on $X$ generated by $\{0, u, v, 1$, Constants $\}$. Then for $w=1-u$ and $\alpha=0.5$, we see that $\left(X, t^{*}\right)$ is $\alpha-\operatorname{SFR}\left(\right.$ iii) and $\left(X, t^{*}\right)$ is $\alpha-\operatorname{SFR}($ iv), but $\left(X, t^{*}\right)$ is not $\alpha-\operatorname{SFR}(\mathrm{ii})$ as they do not exist any $u, v \in t^{*}$ such that $u(x)>\alpha, v(y)=1$, $y \in w^{-1}\{1\}$ and $u \cap v \leq \alpha$.

Example 2.5: Let $X=\{x, y\}$ and $u, v, w \in I^{X}$ are defined by $u(x)=0.9, u(y)=0$, $v(x)=0.5, v(y)=1, w(x)=1, w(y)=0$. Consider the supra fuzzy topology $t^{*}$ on $X$ generated 
by $\{0, u, v, w, 1$, Constants $\}$. Then for $\alpha=0.6$ and $p=1-w$, it is seen that $\left(X, t^{*}\right)$ is $\alpha$-SFR(iv) but $\left(X, t^{*}\right)$ is not $\alpha-\operatorname{SFR}($ iii).

This completes the proof.

Theorem 2.6: If $\alpha, \beta \in t^{*}$ with $0 \leq \alpha \leq \beta<1$, then

(a) $\left(X, t^{*}\right)$ is $\alpha$-SFR(i) implies $\left(X, t^{*}\right)$ is $\beta$ - SFR(i).

(b) $\left(X, t^{*}\right)$ is $\alpha$ - SFR(iii) implies $\left(X, t^{*}\right)$ is $\beta$ - SFR(iii).

Proof. Suppose that $\left(X, t^{*}\right)$ is $\alpha$ - SFR (i). We have to prove that $\left(X, t^{*}\right)$ is $\beta$ - SFR (i). Let $w \in\left(t^{*}\right)^{c}$ and $x \in X$ with $w(x)<1$. Since $\left(X, t^{*}\right)$ is $\alpha$-SFR (i), for $\alpha \in I_{1}$, there exist $u, v \in t^{*}$ such that $u(x)=1, v(y)=1, y \in w^{-1}\{1\}$ and $u \cap v \leq \alpha$. Since $\alpha \leq \beta$, then $u \cap v \leq \beta$. So it is observed that $\left(X, t^{*}\right)$ is $\beta$ - SFR (i).

Example 2.7: Let $X=\{x, y\}$ and $u, v \in I^{X}$ are defined by $u(x)=1, u(y)=0, v(x)=0.7$, $v(y)=1$. Consider the supra fuzzy topology $t^{*}$ on $X$ generated by $\{0, u, v, 1$, constants $\}$. Then for $w=1-u, \alpha=0.8, \beta=0.6$. We see that $\left(X, t^{*}\right)$ is $\beta$-SFR (i) but $\left(X, t^{*}\right)$ is not $\alpha$-SFR(i). In the same way, we can prove that

$$
\left(X, t^{*}\right) \text { is } \alpha \text { - SFR (iii) implies }\left(X, t^{*}\right) \text { is } \beta \text { - SFR (iii). }
$$

Theorem 2.8: Let $\left(X, t^{*}\right)$ be a supra fuzzy topological space and $I_{\alpha}\left(t^{*}\right)=\left\{u^{-1}(\alpha, 1]\right.$ : $\left.u \in t^{*}\right\}$, then $\left(X, t^{*}\right)$ is $0-\operatorname{SFR}(\mathrm{i})$ implies $\left(X, I_{0}\left(t^{*}\right)\right)$ is Regular.

Proof. Suppose $\left(X, t^{*}\right)$ be a 0 -SFR (i). We have to prove that $\left(X, I_{0}\left(t^{*}\right)\right)$ is Regular. Let $V$ be a closed set in $I_{0}\left(t^{*}\right)$ and $x \in X$ such that $x \notin V$. Then $V^{c} \in I_{0}\left(t^{*}\right)$ and $x \in V^{c}$. So, by the definition of $I_{0}\left(t^{*}\right)$, there exists an $u \in t^{*}$ such that $V^{c}=u^{-1}(0,1]$, i.e., $u(x)>0$. Since $u \in t^{*}$, then $u^{c}$ is closed supra fuzzy set in $t^{*}$ and $u^{c}(x)<1$. Since $\left(X, t^{*}\right)$ is $0-\operatorname{SFR}(\mathrm{i})$, there exist $v, w \in t^{*}$ such that $v(x)=1, w \geq 1_{\left(u^{c}\right)^{-1}\{1\}}, v \cap w=0$.

(a) Since $v, w \in t^{*}$ then $v^{-1}(0,1], w^{-1}(0,1] \in I_{0}\left(t^{*}\right)$ and $x \in v^{-1}(0,1]$

(b) Since $w \geq 1_{\left(u^{c}\right)^{-1}\{1\}}$ then $w^{-1}(0,1] \supseteq\left(_{\left(u^{c}\right)^{-1}\{1\}}\right)^{-1}(0,1]$.

(c) And $v \cap w=0$, mean $(v \cap w)^{-1}(0,1]=v^{-1}(0,1] \cap w^{-1}(0,1]=\phi$.

Now, we have

$$
\begin{aligned}
\left(1_{\left(u^{c}\right)^{-1}\{1\}}\right)^{-1}(0,1] & =\left\{x: 1_{\left(u^{c}\right)^{-1}\{1\}}(x) \in(0,1]\right\} \\
& =\left\{x: 1_{\left(u^{c}\right)^{-1}\{1\}}(x)=1\right\} \\
& =\left\{x: x \in\left(u^{c}\right)^{-1}\{1\}\right\} \\
& =\left\{x: u^{c}(x)=1\right\} \\
& =\{x: u(x)=0\} \\
& =\left\{x: x \notin V^{c}\right\} \\
& =\{x: x \in V\} \\
& =V .
\end{aligned}
$$


Put $W=v^{-1}(0,1]$ and $W^{*}=W^{-1}(0,1]$, then $x \in W, W^{*} \supseteq V$ and $W \cap W^{*}=\phi$. Hence it is clear that $\left(X, I_{0}\left(t^{*}\right)\right)$ is Regular.

Example 2.9: Let $X=\{x, y\}, u, v \in I^{X}$, where $u, v$ are defined by $u(x)=0.8, u(y)=0, v(x)=0$ and $v(y)=1$, consider the supra fuzzy topological space $t^{*}$ on $X$ generated by $\{u, v\} \cup\{$ constants $\}$. For $w=1-u$, we see that $\left(X, t^{*}\right)$ is not 0 -SFT(i). Now $I_{0}\left(t^{*}\right)=$ $\{X, \phi,\{x\},\{y\}\}$, here it is clear that $I_{0}\left(t^{*}\right)$ is a supra fuzzy topology on $X$ and hence $\left(X, I_{0}\left(t^{*}\right)\right)$ is a supra regular space.

Theorem 2.10: Let $\left(X, t^{*}\right)$ be a supra fuzzy topological space $A \subseteq X$, and $t^{*}=\left\{u / A: u \in t^{*}\right\}$, then $1_{\left((u / A)^{c}\right)^{-1}\{1\}}(x)=\left(1_{\left(u^{c}\right)^{-1}\{1\}} / A\right)(x)$.

Proof. Let $w$ be a closed supra fuzzy set in $t_{A}^{*}$, i.e., $w \in t_{A}^{*}{ }^{c}$, then $u / A=w^{c}$, where $u \in t^{*}$.

Now, we have

$$
\begin{aligned}
1_{\left((u / A)^{c}\right)^{-1}\{1\}}(x) & =\left\{\begin{array}{lll}
0 & \text { if } & x \notin\left((u / A)^{c}\right)^{-1}\{1\} \\
1 & \text { if } & x \in\left((u / A)^{c}\right)^{-1}\{1\}
\end{array}\right. \\
& =\left\{\begin{array}{lll}
0 & \text { if } & x \notin\left\{y:(u / A)^{c}(y)=1\right\} \\
1 & \text { if } & x \in\left\{y:(u / A)^{c}(y)=1\right\}
\end{array}\right. \\
& =\left\{\begin{array}{lll}
0 & \text { if } & (u / A)^{c}(x)<1 \\
1 & \text { if } & (u / A)^{c}(x)=1
\end{array}\right. \\
& =\left\{\begin{array}{lll}
0 & \text { if } & w(x)<1 \\
1 & \text { if } & w(x)=1
\end{array}\right.
\end{aligned}
$$

$$
\text { Again, } \begin{aligned}
1_{\left(u^{c}\right)^{-1}\{1\}}(x)=\left\{\begin{array}{lll}
0 & \text { if } & x \notin\left(u^{c}\right)^{-1}\{1\} \\
1 & \text { if } & x \in\left(u^{c}\right)^{-1}\{1\}
\end{array}\right. \\
=\left\{\begin{array}{lll}
0 & \text { if } & x \notin\left\{y: u^{c}(y)=1\right\} \\
1 & \text { if } & x \in\left\{y: u^{c}(y)=1\right\}
\end{array}\right. \\
=\left\{\begin{array}{lll}
0 & \text { if } & u^{c}(x)<1 \\
1 & \text { if } & u^{c}(x)=1
\end{array}\right.
\end{aligned}
$$

$$
\text { Now, } \begin{aligned}
\left(1_{\left(u^{c}\right)^{-1}\{1\}} / A\right)(x) & =\left\{\begin{array}{lll}
0 & \text { if } & \left(u^{c} / A\right)(x)<1 \\
1 & \text { if } & \left(u^{c} / A\right)(x)=1
\end{array}\right. \\
& =\left\{\begin{array}{lll}
0 & \text { if } & (u / A)^{c}(x)<1 \\
1 & \text { if } & (u / A)^{c}(x)=1
\end{array}\right.
\end{aligned}
$$




$$
=\left\{\begin{array}{lll}
0 & \text { if } & w(x)<1 \\
1 & \text { if } & w(x)=1
\end{array}\right.
$$

Therefore, we have $1_{\left((u / A)^{c}\right)^{-1}\{1\}}(x)=\left(1_{\left(u^{c}\right)^{-1}\{1\}} / A\right)(x)$.

Theorem 2.11: Let $\left(X, t^{*}\right)$ be a supra fuzzy topological space and $A \subseteq X$ and $t^{*}{ }_{A}=\left\{u / A: u \in t^{*}\right\}$, then

(a) $\left(X, t^{*}\right)$ is $\alpha$-SFR (i) implies $\left(A, t_{A}^{*}\right)$ is $\alpha$-SFR (i).

(b) $\left(X, t^{*}\right)$ is $\alpha$-SFR (ii) implies $\left(A, t_{A}^{*}\right)$ is $\alpha$ - SFR (ii).

(c) $\left(X, t^{*}\right)$ is $\alpha$-SFR (iii) implies $\left(A, t_{A}^{*}\right)$ is $\alpha$ - SFR (iii).

(d) $\left(X, t^{*}\right)$ is $\alpha$-SFR(iv) implies $\left(A, t_{A}^{*}\right)$ is $\alpha$-SFR(iv).

Proof. Let $\left(X, t^{*}\right)$ be $\alpha$-SFR(i). We have to prove that $\left(A, t_{A}^{*}\right)$ is $\alpha$-SFR(i). Let $w$ be a closed fuzzy set in $t_{A}^{*}$, and $x^{*} \in A$ such that $w\left(x^{*}\right)<1$. This implies that $w^{c} \in t_{A}^{*}$ and $w^{c}\left(x^{*}\right)>0$. So there exists a $u \in t^{*}$ such that $u / A=w^{c}$ and clearly $u^{c}$ is closed in $t^{*}$ and $u^{c}\left(x^{*}\right)=(u / A)^{c}\left(x^{*}\right)=w\left(x^{*}\right)<1$, i.e., $u^{c}\left(x^{*}\right)<1$. Since $\left(X, t^{*}\right)$ is $\alpha$-SFR (i), for $\alpha \in I_{1}$, there exist $v, v^{*} \in t^{*}$ such that $v\left(x^{*}\right)=1, v^{*} \geq 1_{\left(u^{c}\right)^{-1}\{1\}}$ and $v \cap v^{*} \leq \alpha$. Since $v, v^{*} \in t^{*}$, then $v / A$, $v^{*} / A \in t_{A}^{*}$ and $v / A\left(x^{*}\right)=1, v^{*} / A \geq\left(1_{\left(u^{c}\right)^{-1}\{1\}} / A\right)$ and $v / A \cap v^{*} / A=\left(v \cap v^{*}\right) / A \leq \alpha$.

But $1_{\left(u^{c}\right)^{-1}\{1\}} / A=1_{\left((u / A)^{c}\right)^{-1}\{1\}}=1_{w^{-1}\{1\}}$, then $v^{*} / A \geq 1_{w^{-1}\{1\}}$. Hence it is clear that $(A$, $t_{A}^{*}$ ) is $\alpha$ - SFR (i).

The proofs of (b), (c) and (d) are similar.

Theorem 2.12: Let $(X, T)$ be a topological space and $T^{*}$ be associated supra topology with $T$. Consider the following statements:

1) $\left(X, T^{*}\right)$ is a Regular space.

2) $\left(X, \omega\left(T^{*}\right)\right)$ is $\alpha$-SFR (i).

3) $\left(X, \omega\left(T^{*}\right)\right)$ is $\alpha$-SFR (ii).

4) $\left(X, \omega\left(T^{*}\right)\right)$ is $\alpha$-SFR (iii).

5) $\left(X, \omega\left(T^{*}\right)\right)$ is $\alpha$-SFR (iv).

Then the following statements are true:

(a) (1) implies (2) implies (3) implies (5) implies (1),

(b) (1) implies (2) implies (4) implies (5) implies (1). 
Proof. First, suppose that $\left(X, T^{*}\right)$ be regular space. We shall prove that $\left(X, \omega\left(T^{*}\right)\right)$ is $\alpha$-SFR (i). Let $w$ be a closed supra fuzzy set in $\omega\left(T^{*}\right)$ and $x \in X$ such that $w(x)<1$, then $w^{c} \in \omega\left(T^{*}\right)$ and $w^{c}(x)>0$. Now we have $\left(w^{c}\right)^{-1}(0,1] \in T^{*}, x \in\left(w^{c}\right)^{-1}(0,1]$. Also it is clear that $\left[\left(w^{c}\right)^{-1}(0,1]\right]^{c}=w^{-1}\{1\}$ be a closed in $T^{*}$ and $x \notin w^{-1}\{1\}$. Since $\left(X, T^{*}\right)$ is Regular, then there exist $V, V^{*} \in T$ such that $x \in V, V^{*} \supseteq w^{-1}\{1\}$ and $V \cap V^{*}=\phi$. But by the definition of lower semi continuous functions $1_{V}, 1_{\mathrm{V}^{*}} \in \omega\left(T^{*}\right)$ and $1_{V}(x)=1$, $1_{\mathrm{V}^{*}} \supseteq 1_{\mathrm{w}^{-1}\{1\}}, 1_{V} \cap 1_{\mathrm{V}^{*}}=1_{V \cap V^{*}}=0$. Put $u=1_{V}$ and $v=1_{\mathrm{V}^{*}}$, then, we have $u(x)=1$, $v \supseteq 1_{\mathrm{w}^{-1}\{1\}}$ and $u \cap v \leq \alpha$. Hence $\left(X, \omega\left(T^{*}\right)\right)$ is $\alpha$ - SFR (i).

We can easily show that (2) implies (3), (3) implies (5), (2) implies (4), (4) implies (5). We therefore prove that (5) implies (1).

Let $\left(X, \omega\left(T^{*}\right)\right)$ be $\alpha$ - SFR (iv). Let $x \in X$ and $V$ be a closed set in $T^{*}$, such that $x \notin V$. This implies that $V^{\mathrm{c}} \in T^{*}$ and $x \in V^{\mathrm{c}}$. But from the definition of $\omega\left(T^{*}\right), 1_{V^{c}} \in \omega\left(T^{*}\right)$, and $\left(1_{V^{c}}\right)^{c}=1_{v}$ closed in $\omega\left(T^{*}\right)$ and $1_{V}(x)=0$. Since $\left(X, \omega\left(T^{*}\right)\right)$ is $\alpha-$ SFR (iv), for $\alpha \in I_{1}$, there exist $u, v \in \omega\left(T^{*}\right)$ such that $u(x)>\alpha, v \geq 1_{\left(1_{V}\right)^{-1}\{1\}}=1_{V}$ and $u \cap v \leq \alpha$. Since $u, v \in \omega\left(T^{*}\right)$, then $u^{-1}(\alpha, 1], v^{-1}(\alpha, 1] \in T^{*}$ and $x \in u^{-1}(\alpha, 1]$. Since $v \geq 1_{V}$, then $v^{-1}(\alpha, 1] \supseteq\left(1_{V}\right)^{-1}(\alpha, 1]=V$, and $u \cap v \leq \alpha$ implies $(u \cap v)^{-1}(\alpha, 1]=u^{-1}(\alpha, 1] \cap v^{-1}(\alpha$, $1]=\phi$. Then by definition, $\left(X, T^{*}\right)$ is Regular space.

Thus it is seen that $\alpha$ - SFR (p) is a good extension of its topological counter part ( $p=$ i , ii , iii, iv ).

Theorem 2.13: Let $\left(X, t^{*}\right)$ and $\left(Y, s^{*}\right)$ be two supra fuzzy topological spaces and $f: X \rightarrow Y$ be continuous, one-one, onto and open map, then

(a) $\left(X, t^{*}\right)$ is $\alpha$ - SFR (i) implies $\left(Y, s^{*}\right)$ is $\alpha$ - SFR (i).

(b) $\left(X, t^{*}\right)$ is $\alpha$ - SFR (ii) implies $\left(Y, s^{*}\right)$ is $\alpha$ - SFR (ii).

(c) $\left(X, t^{*}\right)$ is $\alpha$ - SFR (iii) implies $\left(Y, s^{*}\right)$ is $\alpha$ - SFR (iii).

(d) $\left(X, t^{*}\right)$ is $\alpha$ - SFR (iv) implies $\left(Y, s^{*}\right)$ is $\alpha$ - SFR (iv).

Proof. Suppose $\left(X, t^{*}\right)$ be $\alpha$ - SFR (i). For $w \in\left(s^{*}\right)^{c}$ and $p \in Y$ such that $w(p)<1, f^{-1}(w)$ $\in\left(t^{*}\right)^{c}$ as $f$ is continuous and $x \in X$ such that $f(x)=p$ as $f$ is one-one and onto. Hence $f^{-1}(w)(x)=w(f(x))=w(p)<1$. Since $\left(X, t^{*}\right)$ is $\alpha$ - SFR (i), for $\alpha \in I_{1}$, then there exist $u, v \in t^{*}$ such that $u(x)=1, v(y)=1, y \in\left\{f^{-1}(w)\right\}^{-1}\{1\}$ and $u \cap v \leq \alpha$. This implies that $f(u)(p)=\{\operatorname{Sup} u(x) \quad: f(x)=p\}=1$,

and $f(v) f(y)=\{\operatorname{Sup} v(y)\}=1$ as $f\left(f^{-1}(w)\right) \subseteq w \Rightarrow f(y) \in w^{-1}\{1\}$ and $f(u \cap v) \leq \alpha$ as $u \cap v \leq \alpha \Rightarrow f(u) \cap f(v) \leq \alpha$.

Now, it is clear that for every $f(u), f(v) \in s^{*}$ such that $f(u)(x)=1, f(v)(f(y))=1, f(y)$ $\in w^{-1}\{1\}$ and $f(u) \cap f(v) \leq \alpha$. Hence $\left(Y, s^{*}\right)$ is $\alpha$-SFR (i).

Similarly (b), (c) and (d) can be proved.

Remark: Every homeomorphic image of $\alpha$-regular space is also $\alpha$-regular

Theorem 2.14: Let $\left(X, t^{*}\right)$ and $\left(Y, s^{*}\right)$ be two supra fuzzy topological spaces and $f: X \rightarrow Y$ be a continuous, one-one, onto and closed map then, 
(a) $\left(Y, s^{*}\right)$ is $\alpha$ - SFR (i) implies $\left(X, t^{*}\right)$ is $\alpha$ - SFR (i).

(b) $\left(Y, s^{*}\right)$ is $\alpha$ - SFR (ii) implies $\left(X, t^{*}\right)$ is $\alpha$ - SFR (ii).

(c) $\left(Y, s^{*}\right)$ is $\alpha$ - SFR (iii) implies $\left(X, t^{*}\right)$ is $\alpha$ - SFR (iii).

(d) $\left(Y, s^{*}\right)$ is $\alpha$ - SFR (iv) implies $\left(X, t^{*}\right)$ is $\alpha$ - SFR (iv).

Proof. Suppose $\left(Y, s^{*}\right)$ be $\alpha$ - SFR (i). For $w \in\left(t^{*}\right)^{c}$ and $x \in X$ with $w(x)<1$, then $f(w) \in\left(s^{*}\right)^{c}$ as $f$ is closed and we find $p \in Y$ such that $f(x)=p$ as $f$ is one-one. Now we have $f(w)(p)=\{\operatorname{Sup} w(x): f(x)=p\}<1$. Since $\left(Y, s^{*}\right)$ is $\alpha-$ SFR (i), for $\alpha \in I_{1}$, then there exist $u, v \in s^{*}$ such that $u(f(x))=1, v(y)=1, y \in(f(w))^{-1}\{1\}$ and $u \cap v \leq \alpha$. This implies that $f^{-1}(u), f^{-1}(v) \in t^{*}$ as $f$ is continuous and $u, v \in s^{*}$. Now $f^{-1}(u)(x)=u(f(x))=$ $u(p)=1$ and $f^{-1}(v)(q)=v(f(q))=v(y)=1$ as $f(q)=y, y \in(f(w))^{-1}\{1\}$, i.e., $f(p) \in(f(w))^{-1}\{1\}$ $\Rightarrow q \in w^{-1}\{1\}$ and $f^{-1}(u) \cap f^{-1}(v)=f^{-1}(u \cap v) \leq \alpha$ as $u \cap v \leq \alpha$. Now we observe that there exist $f^{-1}(u), f^{-1}(v) \in t^{*}$ such that $f^{-1}(u)(x)=1, f^{-1}(v)(q)=1, q \in w^{-1}\{1\}$ and $f^{-1}(u) \cap f^{-1}(v) \leq \alpha$. Hence $\left(X, t^{*}\right)$ is $\alpha$-SFR (i).

Similarly, (b), (c) and (d) can be proved.

Remark: Every inverse homeomorphic image of $\alpha$-regular space is also $\alpha$-regular.

Now we recall the following diagrams from refs. [11], [12] and [13], respectively:

(a)

$(X, t)$ is $\alpha-T_{0}$ (i) $(X, t)$ is $\alpha-T_{0}$ (ii)

(b)

$\left(X, t^{*}\right)$ is $\alpha-T_{1}(\mathrm{i})$.

$\left(X, t^{*}\right)$ is $\alpha-T_{1}$ (ii)

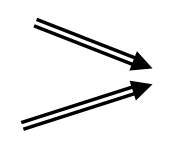

$\left(X, t^{*}\right)$ is $\alpha-T_{1}(\mathrm{iii}) \Rightarrow\left(X, t^{*}\right)$ is $T_{1}(\mathrm{iv})$.

(c)

$(X, t)$ is $\alpha-T_{2}(\mathrm{i})$

$(X, t)$ is $\alpha-T_{2}(\mathrm{ii})$

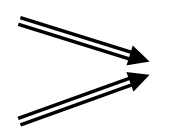

$(X, t)$ is $\alpha-T_{2}(\mathrm{iii})$.

Theorem 2.15: The following are true:

(i) $\left(X, t^{*}\right)$ is an $\alpha-$ SFR (iv) $+\alpha-T_{0}$ (i) $\Rightarrow \alpha-T_{2}$ (i) $\Rightarrow \alpha-T_{1}(\mathrm{i})$.

(ii) $\left(X, t^{*}\right)$ is an $\alpha-$ SFR (iv) $+\alpha-T_{0}\left(\right.$ ii) $\Rightarrow \alpha-T_{2}$ (ii) $\Rightarrow \alpha-T_{1}$ (ii).

Proof: The proof is easy. 
However the arrows are in (i) and (ii) are not reversible

The following examples will serve the purpose.

Example 2.16: Let $X=\{x, y\}$ and $u, v$ be fuzzy sets in $X$, where $u(x)=1, u(y)=0.5, v(x)=$ $0.6, v(y)=1$. Consider the fuzzy supra topology $t^{*}$ on $X$ generated by $\{u, v\} \cup\{$ Constants $\}$. For $w=1-u$, it is clear that $\left(X, t^{*}\right)$ is $\alpha-T_{2}$ (i) but it is not $\square$ - SFR (iv).

Similarly the non reverse civility of (ii) can be shown.

The proof is now complete.

\section{References}

1. L.A.Zadeh, Fuzzy sets. Information and control 8, 338 (1965). http://dx.doi.org/10.1016/S0019-9958(65)90241-X

2. C. L. Chang, J. Math. Anal Appl. 24, 182 (1968). http://dx.doi.org/10.1016/0022-247X(68)90057-7

3. R. Lowen, J. Math. Anal. Appl. 56, 621 (1976). http://dx.doi.org/10.1016/0022-247X(76)90029-9

4. A. S. Mashhour, A. A. Allam, F. S. Mahmoud, and F. H. Khedr, Indian J. Pure and Appl. Math. 14 (4), 502 (1983).

5. M. E. Abd EL-Monsef, and A. E. Ramadan, Indian J. Pure and Appl. Math. 18 (4), 322 (1987).

6. D. M. Ali, Fuzzy Sets and Systems 35, 101 (1990). http://dx.doi.org/10.1016/0165-0114(90)90022-X

7. D. M. Ali, The Journal of Fuzzy Mathematics (Los Angeles) 1 (2), 311 (1993).

8. K. K. Azad, J. Math. Anal. Appl. 82 (1), 14 (1981). http://dx.doi.org/10.1016/0022-247X(81)90222-5

9. P. P. Ming and M. L. Ying. J. Math. Anal. Appl. 77, 20 (1980). http://dx.doi.org/10.1016/0022-247X(80)90258-9

10. C. K. Wong, J. Math. Anal. Appl. 45, 512 (1974). http://dx.doi.org/10.1016/0022-247X(74)90090-0

11. M. S. Hossain and D. M. Ali, J. Math. and Math. Sc. 24, 95 (2009).

12. M. F. Hoque, M. S. Hossain, and D. M. Ali, J. Mech. Cont. Math. Sci. 6 (2), 875 (2012).

13. M. S. Hossain and D. M. Ali, J. Bang. Acad. Sci. 29, 201 (2005). 